\title{
SYMMETRIC SOLUTIONS FOR TWO-BODY DYNAMICS IN A COLLISION PREVENTION MODEL
}

\author{
DAVID J. GATES ${ }^{1}$
}

(Received 19 January, 2010; revised 5 May, 2011)

\begin{abstract}
This paper presents the first analytical solutions for the three-dimensional motion of two idealized mobiles controlled by a particular guidance law designed to avoid a collision with minimal path deviation. The mobiles can be regarded as particles, and guidance can be interpreted as complex forces of interaction between the particles. The motion is then a generalized form of two-body Newtonian dynamics. If the mobiles have equal speeds, the relative motion is determined through various transformations of the differential equations. Solvability relies on congruence and symmetries of the paths, which is exploited to reduce the original twelve first-order differential equations to three firstorder equations for the relative motion. The resulting state space is partitioned into five invariant subsets, with various symmetries and stabilities. One of these sets describes planar motion, where simple explicit solutions are given. In nonplanar motion, the solution is formally reduced to quadrature. A numerical calculation gives the separation at the closest point of approach, which provides control over minimum separation. The results should be of interest because of their application, which includes, most importantly, the prevention of midair collisions between aircraft, but also potential application to land, water and space vehicles. The solutions should be of interest to mathematical specialists in dynamical systems, because of some novel constants of the motion, novel symmetries, and the associated reducibility of the equations.
\end{abstract}

2010 Mathematics subject classification: primary 70-XX; secondary 37-XX.

Keywords and phrases: collision prevention, two-body problem, symmetric solutions.

\section{Introduction}

When a collision between two mobiles is imminent, they could be steered onto safe courses by some form of automated control. This can be achieved by applying suitable command accelerations. For unmanned, robotic vehicles, such control is natural. For manually controlled vehicles, the control could be taken over by the automated system when human control is unpredictable or too difficult. This circumstance is

\footnotetext{
${ }^{1}$ Optimisation in Air Transport Management Team, Mathematics, Informatics and Statistics, Commonwealth Scientific and Industrial Research Organisation, GPO Box 664, Canberra ACT 2601, Australia; e-mail: davidgates@grapevine.com.au.

(c) Australian Mathematical Society 2011, Serial-fee code 1446-1811/2011\$16.00
} 
especially relevant for aircraft in danger of a midair collision. Experimental systems with this purpose $[14,16]$ have been developed for small jet aircraft, and preliminary tests carried out. Such methods might also be applied to other mobiles such as spacecraft, surface vehicles, ships or submersibles. Each mobile needs continuous, accurate information about the positions and velocities of both mobiles, which could be provided by on-board sensors, inertial navigation systems, the Global Positioning System and data links between mobiles [8].

There is a long history of computer simulation studies involving different forms of command accelerations [5, 9]. The functional forms of the accelerations define the guidance law or guidance logic. Early studies were based on Coulomb repulsion, but this proved unsatisfactory, mainly because it produced impractically large accelerations. Other vector fields, including magnetic fields and vortex fields, showed some promise. These have the advantage of allowing the paths of two mobiles to cross, thereby avoiding excessive accelerations.

The guidance logic studied here achieves crossing paths in a natural way, and appears to be quite effective. It was introduced by Gazit and Powell [8] and Zeghal [18], and further studied by the author [7]. Some exact solutions for this logic, in planar motion, were also given by the author [7]. The present paper provides the first exact solution for nonplanar, three-dimensional (3D) motion of mobiles with equal speeds. Much of the paper is devoted to developing the solution method and classifying the types of motions from a dynamical systems viewpoint. Practical matters, such as turning capabilities and the achievement of a safe separation between mobiles, are also studied.

The equations of motion are given in Section 2, and the solution strategy outlined in Section 3. The system is transformed in terms of other vectors in Section 4. Component equations relative to a rotating frame of reference are derived in Section 5. Three first-order equations for the relative motion are obtained in Section 6. General properties of the motion and paths are deduced in Section 7. Simple solutions for planar (2D) motion are provided in Section 8. Nonplanar (3D) solutions are given in Section 9 and invariant sets are identified. The 3D motion is illustrated in Section 10. Control of the closeness of approach is described in Section 11. Symmetric initial conditions, which generate an invariant set, are described in Section 12. A discussion is contained in Section 13.

\section{Equations of motion}

The following system of differential equations has application to a dynamically efficient method for preventing a collision between two mobiles, such as aircraft. We consider the 3D motion of two interacting, point particles of unit mass, determined by the Newtonian equations

$$
\dot{\boldsymbol{r}}_{i}=\boldsymbol{V}_{i}, \quad \dot{\boldsymbol{V}}_{i}=\boldsymbol{a}_{i},
$$

for $i=1,2$, where $\boldsymbol{r}_{i}(t)$ and $\boldsymbol{V}_{i}(t)$ are the position vector and velocity vector of particle $i$ at time $t$, the over-dot indicates a time derivative, and the accelerations 


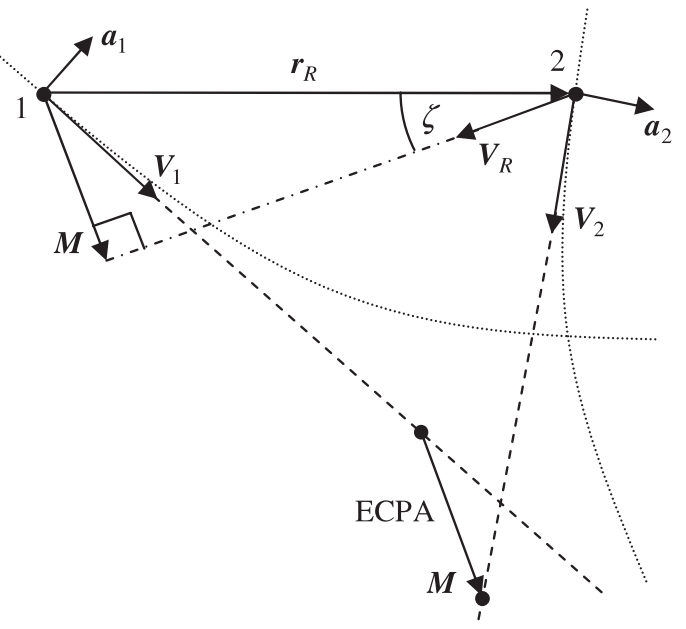

FigurE 1. A sketch of particle paths (dotted lines) and relevant vectors. The dashed line shows straight paths defining the ECPA. The dash-dotted line shows the construction of $\boldsymbol{M}$.

have the form

$$
\begin{aligned}
& \boldsymbol{a}_{1}=-f \overline{\boldsymbol{m}}_{1}, \\
& \boldsymbol{a}_{2}=f \overline{\boldsymbol{m}}_{2},
\end{aligned}
$$

with force coefficient $f\left(r_{R}\right)>0$, where $r_{R}=\left|\boldsymbol{r}_{R}\right|$ and $\boldsymbol{r}_{R}=\boldsymbol{r}_{2}-\boldsymbol{r}_{1}$ is the current relative position vector; various functional forms of $f$ will be considered. Also

$$
\overline{\boldsymbol{m}}_{i}=\boldsymbol{u}_{i} \times \boldsymbol{m} \times \boldsymbol{u}_{i},
$$

where $\boldsymbol{u}_{i}$ is the unit vector parallel to $\boldsymbol{V}_{i}$, and $\boldsymbol{m}$ is the unit vector parallel to the expected (or extrapolated) miss vector

$$
\boldsymbol{M}= \begin{cases}\boldsymbol{w}_{R} \times \boldsymbol{r}_{R} \times \boldsymbol{w}_{R} & \text { if } \boldsymbol{V}_{1} \neq \boldsymbol{V}_{2} \\ \boldsymbol{r}_{R} & \text { otherwise, }\end{cases}
$$

where $\boldsymbol{w}_{R}$ is the unit vector along the relative velocity

$$
\boldsymbol{V}_{R}=\dot{\boldsymbol{r}}_{R}=\boldsymbol{V}_{2}-\boldsymbol{V}_{1} \text {. }
$$

Thus $\boldsymbol{M}$ is the component of $\boldsymbol{r}_{R}$ normal to $\boldsymbol{V}_{R}$, and $\overline{\boldsymbol{m}}_{i}$ is the component of $\boldsymbol{m}$ normal to $\boldsymbol{u}_{i}$. Particle paths and relevant vectors are illustrated in Figure 1.

If $\boldsymbol{M}=\mathbf{0}$ then $\boldsymbol{m}$ is not defined. If $\boldsymbol{M}$ falls to zero during the motion, we define $\boldsymbol{a}_{i}=\mathbf{0}$; this can happen, as shown in Theorem 7.5. If $\boldsymbol{M}=\mathbf{0}$ initially, for converging particles, then the motion is indeterminate. Then a suitable rule can be applied [7] to initiate motion, but this case is not considered here.

The meaning of $\boldsymbol{M}$ is as follows. If two particles are on convergent paths and were to continue with their current velocity vectors, they would reach a configuration of least separation $r_{R}$, called the expected (or extrapolated) closest point of approach (ECPA), 
as shown in Figure 1. Then $\boldsymbol{M}$ is the 3D vector directed from particle 1 to particle 2 at the ECPA. One can obtain $\boldsymbol{M}$ by viewing the motion from a frame moving with particle 1 . Then particle 2 appears to have velocity vector $\boldsymbol{V}_{R}$. Its extrapolated straight path, shown in Figure 1, is closest to particle 1 at the normal $\boldsymbol{M}$ through particle 1; hence (2.5) holds.

The accelerations are formulated with the purpose of increasing the expected missdistance (EMD) $M=|\boldsymbol{M}|$. It turns out that this causes the particles (mobiles) to evade each other without excessive turning $[7,18]$. For example, in planar motion it allows the paths to cross.

As $\boldsymbol{a}_{i}$ is normal to $\boldsymbol{V}_{i}$, each particle travels at constant speed; that is, the speeds are constants of the motion. Hence the forces do no work (like magnetic forces) and there is no energy principle. The forces of interaction between the particles are evidently quite complex, involving quintuple vector products originating from (2.4) and (2.5). Like magnetic forces (the Biot-Savart law) they are not "central forces". Moreover, $\boldsymbol{a}_{1} \neq-\boldsymbol{a}_{2}$ in general, in violation of Newton's third law, and so the centre of mass of the particles does not have a constant velocity vector. The effective force between particles is not always repulsive [7]. The motion is not planar in general, but essentially threedimensional. Galilean invariance does not hold because absolute velocities appear in the $\overline{\boldsymbol{m}}_{i}$. These unusual features imply that the dynamics has little in common with typical two-body dynamics.

\section{Solution strategy}

Equations (2.1) comprise twelve first-order differential equations in twelve state variables: the three components of each of the vectors $\boldsymbol{r}_{1}, \boldsymbol{r}_{2}, \boldsymbol{V}_{1}$ and $\boldsymbol{V}_{2}$. The method of solution proceeds by reducing the number of equations through various transformations. This is achieved by exploiting various symmetries, that is, the invariance of the system of equations under various groups of transformations of the state variables, as follows.

(a) The system is invariant under $E^{+}(3)$, the Euclidean group of proper 3D rotations (excluding reflections).

(b) The system is $T$-invariant (time-reversal).

(c) The system is invariant if the labels 1 and 2 are exchanged. The particles are said to be interchangeable.

(d) The initial speeds $V$ are chosen to be the same. The equations imply that these speeds are maintained: $\left|\boldsymbol{V}_{1}\right|=\left|\boldsymbol{V}_{2}\right|=V$.

(e) The system has a scale invariance for suitable $f$.

(f) There are other implicit symmetries associated with constants of the motion.

The restrictions (c) and (d) are limiting from a practical point of view, but have potential application if the mobiles are similar and speeds are set by regulation, convention or linked controls.

A continuous symmetry may be associated with a first integral or constant of the motion, leading to a transformation which reduces the order of a system. This 
principle is extensively applied to dynamical systems [1, 2, 6, 12, 17], continuum mechanics [10, 13] and other mechanical systems [3] including robotics. There are formal methods of obtaining the transformations from generators of groups [11], but in practice it is often easier to infer the transformations more intuitively, and this is the approach used here.

We do not attempt to give a complete analytical solution of the system. However, it is notable and surprising that aspects of the relative motion can be described by a system of three first-order equations. This system has some novel constants of the motion and, surprisingly, its solution reduces to quadrature. Very few dynamical systems are thus reducible, and so it might it be interesting to pursue the more formal approach in the future.

\section{Transformed vector differential equations}

Here the differential equations (2.1) are transformed in terms of the relative and mean motions. There are two reasons for this. First, the relative motion and the variation of the particle separation $r_{R}$ over time are of central interest, because one has a dangerous situation when $r_{R}$ becomes small. Second, it provides a significant reduction of the original system (2.1).

The centre of mass, or centroid, has position vector $\overline{\boldsymbol{r}}=\left(\boldsymbol{r}_{1}+\boldsymbol{r}_{2}\right) / 2$ and velocity vector $\bar{V}=\left(\boldsymbol{V}_{1}+\boldsymbol{V}_{2}\right) / 2$. We assume that $M \neq 0$ in the following. The transformed equations are

$$
\begin{aligned}
\dot{\boldsymbol{r}}_{R} & =\boldsymbol{V}_{R}, \quad \dot{\overline{\boldsymbol{r}}}=\overline{\boldsymbol{V}}, \\
\dot{\boldsymbol{V}}_{R} & =2 f(\boldsymbol{m}-\chi \overline{\boldsymbol{V}}), \\
\dot{\overline{\boldsymbol{V}}} & =-\frac{1}{2} f \chi \boldsymbol{V}_{R},
\end{aligned}
$$

where

$$
\chi=\frac{\boldsymbol{m} \cdot \overline{\boldsymbol{V}}}{V^{2}}=\frac{\boldsymbol{r}_{R} \cdot \overline{\boldsymbol{V}}}{M V^{2}} .
$$

This system is closed because $M$ and $\boldsymbol{m}$ depend only on $\boldsymbol{r}_{R}$ and $\boldsymbol{V}_{R}$. Equation (4.1) is obtained as follows. Equations (2.1)-(2.3) imply

$$
\dot{\boldsymbol{V}}_{R}=f\left(\overline{\boldsymbol{m}}_{1}+\overline{\boldsymbol{m}}_{2}\right)=f\left(2 \boldsymbol{m}-\boldsymbol{m} \cdot \boldsymbol{u}_{1} \boldsymbol{u}_{1}-\boldsymbol{m} \cdot \boldsymbol{u}_{2} \boldsymbol{u}_{2}\right) .
$$

In general $\boldsymbol{m} \cdot \boldsymbol{V}_{R}=0$, which here implies, as $\left|\boldsymbol{V}_{1}\right|=\left|\boldsymbol{V}_{2}\right|$,

$$
\boldsymbol{m} \cdot \boldsymbol{u}_{1}=\boldsymbol{m} \cdot \boldsymbol{u}_{2}=\boldsymbol{m} \cdot \overline{\boldsymbol{u}}
$$

where

$$
\overline{\boldsymbol{u}}=\frac{1}{2}\left(\boldsymbol{u}_{1}+\boldsymbol{u}_{2}\right)
$$

This implies

$$
\dot{\boldsymbol{V}}_{R}=2 f(\boldsymbol{m}-\boldsymbol{m} \cdot \overline{\boldsymbol{u}} \overline{\boldsymbol{u}}),
$$


which is (4.1). From (2.1)-(2.3),

$$
\dot{\overline{\boldsymbol{V}}}=\frac{1}{2} f\left(\overline{\boldsymbol{m}}_{2}-\overline{\boldsymbol{m}}_{1}\right)=\frac{1}{2} f\left(\boldsymbol{m} \cdot \boldsymbol{u}_{1} \boldsymbol{u}_{1}-\boldsymbol{m} \cdot \boldsymbol{u}_{2} \boldsymbol{u}_{2}\right) .
$$

Then (4.4) yields (4.2). Moreover, (4.1) and (4.2) imply, with $\bar{V}=|\overline{\boldsymbol{V}}|$,

$$
\begin{aligned}
\frac{d}{d t}\left(\boldsymbol{V}_{R} \cdot \overline{\boldsymbol{V}}\right) & =\dot{\boldsymbol{V}}_{R} \cdot \overline{\boldsymbol{V}}+\boldsymbol{V}_{R} \cdot \dot{\overline{\boldsymbol{V}}} \\
& =2 f\left(\boldsymbol{m} \cdot \overline{\boldsymbol{V}}-\chi \bar{V}^{2}\right)-\frac{1}{2} f \chi V_{R}^{2} \\
& =2 f\left(\boldsymbol{m} \cdot \overline{\boldsymbol{V}}-\chi V^{2}\right),
\end{aligned}
$$

which is zero by (4.3). Thus the new equations, and the initial orthogonality, imply that $\boldsymbol{V}_{R}$ and $\overline{\boldsymbol{V}}$ remain orthogonal, and so this need not be imposed as an additional constraint. The orthogonality removes one degree of freedom and is exploited in (5.9) for reducing the system. The system has an unusual and remarkable property.

Theorem 4.1. $\bar{V}$ is a constant of the motion. As $V_{R}=2 \sqrt{V^{2}-\bar{V}^{2}}$, it is also constant. Equivalently, the angle $\phi_{R}$ between $\boldsymbol{V}_{1}$ and $\boldsymbol{V}_{2}$ is constant.

Proof. Noting that $\bar{V} \dot{\bar{V}}=\overline{\boldsymbol{V}} \cdot \dot{\overline{\boldsymbol{V}}}$ and for equal speeds $\overline{\boldsymbol{V}} \cdot \boldsymbol{V}_{R}=0$, it follows directly from (4.2) that $\bar{V}$ is constant.

Remarks. Then each of (4.1) and (4.2) represents only two scalar equations. For 2D motion, a constant $\phi_{R}$ implies that the two particles turn at the same rate at any instant of time. In $3 \mathrm{D}$ motion, a constant $\phi_{R}$ means that, as viewed by one particle (which sees its path as a fixed axis), the other particle follows a helix of general type [15]. As mentioned, $\overline{\boldsymbol{V}}$ is not constant, because Newton's third law does not hold.

\section{Components of acceleration in a rotating frame}

This section is devoted to expressing equations (4.1) and (4.2) in component form in terms of suitable coordinates, referred to a rotating frame of reference. The method is motivated by the mentioned symmetries, but formal justifications of the choices are not given here.

A convenient frame $F$ has its origin on particle 1, its $x$ axis along $\boldsymbol{r}_{R}$, its $z$ axis along $\boldsymbol{r}_{R} \times \boldsymbol{V}_{R}$, and its $y$ axis along $\boldsymbol{r}_{R} \times \boldsymbol{V}_{R} \times \boldsymbol{r}_{R}$, as shown in Figure 2. Thus the $y$ axis lies in the plane of $\boldsymbol{r}_{R}$ and $\boldsymbol{V}_{R}$. The corresponding orthonormal basis vectors are denoted by $\boldsymbol{e}_{X}, \boldsymbol{e}_{Y}$ and $\boldsymbol{e}_{Z}$. In the following, components of vectors are referred to $F$. Then $\boldsymbol{r}_{R}=r_{R}(1,0,0)$ and

$$
\boldsymbol{V}_{R}=V_{R}(-\cos \zeta, \sin \zeta, 0),
$$

where $\zeta$ is the angle between $\boldsymbol{r}_{R}$ and $-\boldsymbol{V}_{R}$, as shown in Figures 1 and 2. The geometry indicates that $0<\zeta<\pi / 2$ while the particles are closing, and $\pi / 2<\zeta<\pi$ while they are receding. We find in Section 6 that $\zeta$ increases. The angular velocity vector of $\boldsymbol{r}_{R}$ 
has magnitude equal to the rotation rate, and direction along the axis of rotation in the right-hand sense. It can be expressed as

$$
\boldsymbol{\Omega}_{R}=\frac{\boldsymbol{r}_{R} \times \boldsymbol{V}_{R}}{r_{R}^{2}}=(0,0, W \sin \zeta),
$$

where $W=V_{R} / r_{R}$ (Figure 2). Since $F$ is rotating, the time derivative of any vector $\boldsymbol{h}=\left(h_{X}, h_{Y}, h_{Z}\right)$ is

$$
\dot{\boldsymbol{h}}=\left(\dot{h}_{X}, \dot{h}_{Y}, \dot{h}_{Z}\right)+\boldsymbol{\Omega}_{F} \times \boldsymbol{h},
$$

where $\boldsymbol{\Omega}_{F}$ is the angular velocity vector of $F$. Recall that $\boldsymbol{\Omega}_{F}$ is parallel to the axis of rotation of the frame. Since $\boldsymbol{e}_{X}$ is fixed in $F, \dot{\boldsymbol{e}}_{X}=\boldsymbol{\Omega}_{F} \times \boldsymbol{e}_{X}$. Also $\boldsymbol{\Omega}_{R}=\boldsymbol{e}_{X} \times \dot{\boldsymbol{e}}_{X}$ and so

$$
\boldsymbol{\Omega}_{R}=\boldsymbol{e}_{X} \times \boldsymbol{\Omega}_{F} \times \boldsymbol{e}_{X}=\boldsymbol{\Omega}_{F}-Q \boldsymbol{e}_{X},
$$

where $Q=\boldsymbol{\Omega}_{F} \cdot \boldsymbol{e}_{X}$ is the $X$ component of $\boldsymbol{\Omega}_{F}$. One notes the distinction between the angular velocity of a vector and the angular velocity of a frame (or a rigid body), exemplified by (5.4). Only vectors that are normal to $\boldsymbol{\Omega}_{F}$, like the spokes of a spinning wheel, have angular velocity $\boldsymbol{\Omega}_{F}$, the $y$ axis being an example. By contrast, a vector directed along the axis of the wheel (parallel to $\boldsymbol{\Omega}_{F}$ ) has zero angular velocity. Now (5.2) and (5.4) give

$$
\boldsymbol{\Omega}_{F}=(Q, 0, W \sin \zeta),
$$

with $Q$ to be determined. Recalling that $V_{R}$ is constant, one gets from (5.1), (5.3) and (5.5)

$$
\dot{\boldsymbol{V}}_{R}=V_{R} \dot{\zeta}(\sin \zeta, \cos \zeta, 0)+V_{R} \sin \zeta(-W \sin \zeta,-W \cos \zeta, Q)
$$

An expression for the right side of (4.1) is required. First one uses (2.5) and (5.2) to get

$$
\boldsymbol{M}=\frac{r_{R}^{2}}{V_{R}^{2}} \boldsymbol{V}_{R} \times \boldsymbol{\Omega}_{R}=r_{R} \sin \zeta(\sin \zeta, \cos \zeta, 0) .
$$

Assuming the particles are not on a collision course $(\zeta \neq 0$ and $M \neq 0)$, we have

$$
\boldsymbol{m}=s(\sin \zeta, \cos \zeta, 0),
$$

where $s=\operatorname{sgn} \zeta$. Second, $\overline{\boldsymbol{V}} \perp \boldsymbol{V}_{R}$, and so one can write

$$
\overline{\boldsymbol{V}}=\bar{V}(\sin \alpha \sin \zeta, \sin \alpha \cos \zeta, \cos \alpha),
$$

which makes an angle $\alpha$ with the $z$ axis (Figure 2). Thus $\zeta$ and $\alpha$ are the spherical polar angles of $\overline{\boldsymbol{V}}$ relative to axes $y$ and $z$. It turns out (see (6.1)-(6.3)) that $r_{R}, \zeta$ and $\alpha$ suffice as state variables for a description of the relative motion. The last two equations imply $\boldsymbol{m} \cdot \overline{\boldsymbol{V}}=\bar{V} s \sin \alpha$. Then the right side of (4.1) becomes

$$
2 f s(\sin \zeta, \cos \zeta, 0)-2 f \eta^{2} \sin \alpha(\sin \alpha \sin \zeta, \sin \alpha \cos \zeta, \cos \alpha),
$$

where

$$
\eta=\frac{\bar{V}}{V}=\cos \frac{\phi_{R}}{2}<1
$$


is a constant. Equating $X$ components in (5.6) and (5.10) gives

$$
V_{R} \dot{\zeta}-V_{R} W \sin \zeta=2 f s\left(1-\eta^{2} \sin ^{2} \alpha\right)
$$

or $\sin \zeta=0$. There is no solution with $\sin \zeta=0$ for all time, implying $M=0$. Equating $Y$ components in (5.6) and (5.10) gives the same again or $\cos \zeta=0$. There is no solution with $\cos \zeta=0$ for all time, implying constant $r_{R}$. Equating the $Z$ components gives

$$
Q V_{R} \sin \zeta=-2 f \eta^{2} s \sin \alpha \cos \alpha .
$$

A further equation is needed to determine $Q$, and this can be obtained from (4.2). The right side of (4.2) becomes

$$
\frac{f V_{R} \bar{V}}{2 V^{2}} s \sin \alpha(\cos \zeta,-\sin \zeta, 0) .
$$

Writing $\overline{\boldsymbol{V}}=\left(\bar{V}_{X}, \bar{V}_{Y}, \bar{V}_{Z}\right)$ and using (5.3) one has

$$
\dot{\overline{\boldsymbol{V}}}=\left(\dot{\bar{V}}_{X}, \dot{\bar{V}}_{Y}, \dot{\bar{V}}_{Z}\right)+\boldsymbol{\Omega}_{F} \times \overline{\boldsymbol{V}}
$$

Recalling that $\bar{V}$ is constant, one gets from (5.5) and (5.9) the $Z$ component

$$
(\dot{\bar{V}})_{Z}=-\bar{V} \dot{\alpha} \sin \alpha+\bar{V} Q \sin \alpha \cos \zeta
$$

From (5.14), this is zero, and so

$$
Q=\frac{\dot{\alpha}}{\cos \zeta},
$$

or $\sin \alpha=0$. The latter represents a more symmetric solution, as described in Section 12. The $X$ and $Y$ components of (4.2) reproduce existing equations. This redundancy is a manifestation of symmetries.

\section{Reduced equations for the relative motion}

The above equations now combine to produce a reduced system of differential equations for the relative motion. Putting (5.17) in (5.13) gives

$$
\dot{\alpha}=-\frac{2 f \eta^{2} \cos \zeta}{V_{R} \sin \zeta} s \sin \alpha \cos \alpha .
$$

Rearranging (5.12) gives

$$
\dot{\zeta}=\frac{2 f}{V_{R}} s\left(1-\eta^{2} \sin ^{2} \alpha\right)+\frac{V_{R}}{r_{R}} \sin \zeta .
$$

Since $\dot{r}_{R}=\boldsymbol{r}_{R} \cdot \boldsymbol{V}_{R} / r_{R}$, one gets from (5.1)

$$
\dot{r}_{R}=-V_{R} \cos \zeta .
$$

Equations (6.1)-(6.3) determine $\alpha, \zeta$ and $r_{R}$ from their initial values. If the particles are initially converging then $0 \leq \zeta<\pi / 2$, and so $s=1$. Then (6.2) implies that $\zeta$ always increases, and so $s=1$ continues to hold. Thus $s$ is omitted from the equations henceforth. The equations are autonomous, and so they can of course be reduced to two equations by eliminating $t$. 


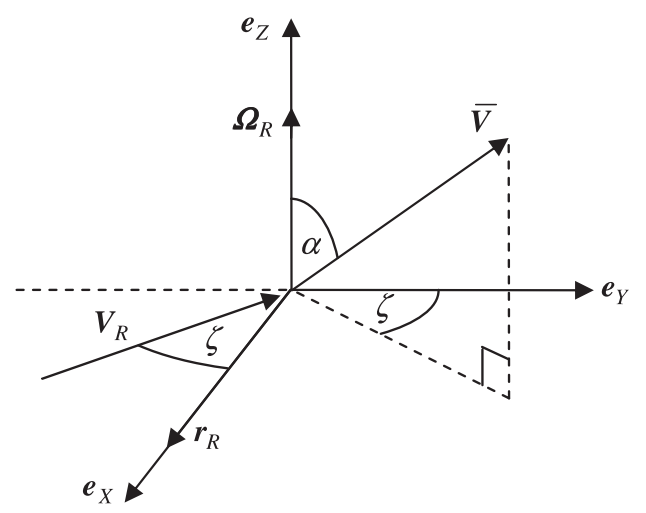

FIGURE 2. The frame $F$ with basis vectors $\boldsymbol{e}_{X}, \boldsymbol{e}_{Y}$ and $\boldsymbol{e}_{Z}$, and other principal angles and vectors.

\section{General properties of the motion}

General properties of the motion can be deduced without solving (6.1)-(6.3). The configuration of smallest particle separation is called the closest point of approach (CPA) (as opposed to the ECPA). It occurs when $\dot{r}_{R}=0$, and so $\zeta=\pi / 2$. Thus $\boldsymbol{r}_{R}, \boldsymbol{V}_{R}$ and $\boldsymbol{\Omega}_{R}$ are mutually orthogonal at CPA (see Figure 2). Let $\bar{t}$ denote the time at CPA.

Definition 7.1. A function $\psi(t)$ that satisfies $\psi(t)=\psi(2 \bar{t}-t)$ is said to be symmetric about CPA. If $\psi(t)=-\psi(2 \bar{t}-t)$ then $\psi$ is said to be antisymmetric about CPA.

THEOREM 7.2. The functions $r_{R}(t), \alpha(t)$ and $M(t)$ are symmetric about CPA, whereas $\zeta(t)-\pi / 2$ is antisymmetric about CPA.

Proof. If $\zeta$ is replaced by $\pi-\zeta$, then $\dot{\alpha}$ becomes $-\dot{\alpha}$ in (6.1), $\dot{\zeta}$ is unchanged in (6.2), and $\dot{r}_{R}$ becomes $-\dot{r}_{R}$ in (6.3). Thus, for every solution $\alpha, \zeta$ and $r_{R}$ with rates $\dot{\alpha}, \dot{\zeta}$ and $\dot{r}_{R}$ before CPA, there is a solution $\alpha, \pi-\zeta$ and $r_{R}$ with rates $-\dot{\alpha}, \dot{\zeta}$ and $-\dot{r}_{R}$ after CPA. From (5.7),

$$
M=r_{R} \sin \zeta
$$

which implies the symmetry of $M(t)$.

THEOREM 7.3. The separation $r_{R}(t)$ is a convex function of $t$.

Proof. Differentiating (6.3) and using (6.2) gives

$$
\ddot{r}_{R}=\dot{\zeta} V_{R} \sin \zeta=2 f\left(1-\eta^{2} \sin ^{2} \alpha\right) \sin \zeta+\frac{V_{R}^{2}}{r_{R}} \sin ^{2} \zeta,
$$

which is positive because $0<\zeta<\pi$.

REMARK. It follows that $r_{R}(t)$ has a unique local minimum, namely CPA. This is of practical significance, because a minimal value of separation is a vital indicator of 
safety. Moreover, $\ddot{r}_{R}=-\dot{v}_{C}$ where $v_{C}$ is the closing speed. Thus the closing speed decreases, which is very desirable behaviour.

We impose two general conditions on $f$.

(a) $f$ is a nonincreasing function of $r_{R}$. This is realistic in applications, because mutual evasion should not be stronger at greater separations. However, a constant $f$ is admitted.

(b) $f$ is strictly positive. This excludes the trivial case where $f=0$ beyond some value of $r_{R}$, where the subsequent motion would be that of free particles.

Theorem 7.4. (a) $M$ increases while $r_{R}$ decreases, and (b) $M$ decreases while $r_{R}$ increases.

Proof. For 3D motion, (6.2), (6.3) and (7.1) imply

$$
\dot{M}=-\frac{2 f r_{R} \dot{r}_{R}}{V_{R}^{2}}\left(1-\eta^{2} \sin ^{2} \alpha\right) .
$$

Then (5.11) implies the theorem.

Remark. Case (a) refers to the situation before CPA, and shows that EMD guidance achieves its basic objective of increasing $M$. In case (b) the particles are receding, and so a decreasing $M$ indicates an improving situation.

The asymptotic behaviour of $M$ as $t \rightarrow \infty$ is related to the asymptotic behaviour of $f$ as $r_{R} \rightarrow \infty$, as follows.

THEOREM 7.5. If $f r_{R}$ is uniformly bounded away from zero then (after CPA) $M$ falls to zero in a finite time.

Proof. In general, $V_{R} \leq 2 V$. By Theorem 7.3, $\dot{r}_{R} \geq \varepsilon>0$ for all $t \geq t(\varepsilon)>\bar{t}$. Then (7.2) and (5.11) imply $\dot{M}<-C$, where

$$
C=\frac{f r_{R} \varepsilon}{V}\left(1-\eta^{2}\right)>0
$$

Thus $0 \leq M<M(\bar{t})-C(t-\bar{t})$. The upper bound is negative for $t>\bar{t}+M(\bar{t}) / C$. Hence $M$ falls to zero at an earlier time.

Theorem 7.6. If $f r_{R} \rightarrow 0$ as $r_{R} \rightarrow \infty$ then $\dot{M} \rightarrow 0$ as $t \rightarrow \infty$.

PRoof. Differentiating $\boldsymbol{r}_{R} \cdot \boldsymbol{r}_{R}=r_{R}^{2}$ gives $\boldsymbol{V}_{R} \cdot \boldsymbol{r}_{R}=\dot{r}_{R} r_{R}$. By Theorem 7.3, for all $t \geq t(\varepsilon)>\bar{t}$, we have $V_{R} \geq \boldsymbol{V}_{R} \cdot \boldsymbol{r}_{R} / r_{R}=\dot{r}_{R}>\varepsilon$. Then (7.2) and Theorem 7.4 give $-2 f r_{R} / \varepsilon<\dot{M}<0$, which completes the proof.

Remark. An unsolved problem is to find conditions on $f$ which imply that $M \rightarrow 0$ as $t \rightarrow \infty$.

REMARK. In the regime of Theorem 7.5, the time $t_{U}$ at which $M$ falls to zero is called the ultimate point of escape (UPE). As mentioned after (2.6), the accelerations are 
thereafter defined as zero, and so particles recede along straight paths, radiating from a past 'collision' point. This is an unusual feature of the motion for a system in which the interaction appears to have infinite range. The $T$-invariance of the equations of motion then implies that there is an earlier time $t_{E}=2 \bar{t}-t_{U}$ when $M=0$ and the accelerations are undefined. This is called the earliest point of guidance (EPG). Equation (7.1) shows that $\zeta=0$ at EPG, $\zeta=\pi / 2$ at $\mathrm{CPA}$, and $\zeta=\pi$ at UPE.

The following property is unusual and striking.

THEOREM 7.7. The curvatures of the paths of the two particles are equal at any instant of time, and are symmetric about CPA. Equivalently, the magnitudes of the two accelerations are equal and symmetric about CPA.

Proof. The curvature of the path of particle $i$ at time $t$ is $\kappa_{i}(t)=\left|\dot{\boldsymbol{V}}_{i}\right| / V^{2}$. From (2.1) and (2.2),

$$
\left|\dot{\boldsymbol{V}}_{i}\right|=f\left|\boldsymbol{m} \times \boldsymbol{u}_{i}\right|=f \sqrt{1-\left(\boldsymbol{m} \cdot \boldsymbol{u}_{i}\right)^{2}} .
$$

From (4.4),

$$
\kappa_{1}=\kappa_{2}=\frac{f}{V^{2}} \sqrt{1-(\boldsymbol{m} \cdot \overline{\boldsymbol{u}})^{2}}=\frac{f}{V^{2}} \sqrt{1-\cos ^{2}\left(\phi_{R} / 2\right) \sin ^{2} \alpha},
$$

where equations (5.8) and (5.9) have been used. Theorem 7.2 then implies that $\kappa_{i}$ is symmetric about CPA.

COROllary 7.8. In planar motion, the paths of the two particles are congruent in $E^{+}(2)$, the Euclidean group of proper $2 D$ rotations. The segment of a path beyond CPA is the mirror image of the segment before CPA.

Proof. A planar curve is completely defined by its sequence of curvatures, and so the paths inherit the properties from Theorem 7.7. The equalities of the curvatures are also implied by Theorem 4.1 .

Theorem 7.9. The torsions $\tau_{i}(t)$ of the two paths satisfy $\tau_{1}(t)=\tau_{2}(2 \bar{t}-t)$.

Proof. As shown in the Appendix, the torsions are given by the expressions

$$
\tau_{i}=\frac{f^{2} J_{i}}{V^{6} \kappa_{i}^{2}},
$$

where

$$
\begin{gathered}
J_{1}=\bar{J}-\frac{1}{2} J_{R}, \quad J_{2}=\bar{J}+\frac{1}{2} J_{R}, \\
\bar{J}=-\left(2 f \bar{V} / V_{R}\right)\left(1-\eta^{2} \sin ^{2} \alpha\right) \cos \alpha
\end{gathered}
$$

and

$$
J_{R}=-V_{R} \dot{\alpha} .
$$

With $t^{*}=2 \bar{t}-t$, Theorem 7.2 implies that $\bar{J}(t)=\bar{J}\left(t^{*}\right)$ and $J_{R}(t)=-J_{R}\left(t^{*}\right)$ because $\dot{\alpha}$ is antisymmetric about CPA. These imply that $J_{1}(t)=J_{2}\left(t^{*}\right)$. Then Theorem 7.7 and (7.4) imply Theorem 7.9. 
Remark. The $\tau_{i}$ are not symmetric about CPA. At UPE and EPG, where $\alpha=\pi / 2$, the $\tau_{i}$ are zero.

Corollary 7.10. The paths of the two particles are congruent in $E^{+}(3)$. When the paths are brought into coincidence, the forward motion on one path coincides with the reversed motion on the other.

PRoof. The shape of a 3D curve is completely defined by its sequence of curvatures and torsions, and so the paths inherit the properties from Theorems 7.7 and 7.9.

Of special interest are force coefficients of the form

$$
f=f_{p} \equiv \frac{p V^{2}}{r_{R}},
$$

where $p>0$ is a dimensionless gain constant. This $f$ is just within the class covered by Theorem 7.5 . We consider the scale transformation

$$
S:\left(\boldsymbol{r}_{1}, \boldsymbol{r}_{2}, \boldsymbol{V}_{1}, \boldsymbol{V}_{2}, t\right) \mapsto\left(\boldsymbol{r}_{1}, \boldsymbol{r}_{2}, \mu \boldsymbol{V}_{1}, \mu \boldsymbol{V}_{2}, t / \mu\right)
$$

for constant $\mu>0$. Then we have the following theorem.

THEOREM 7.11. If $f=f_{p}$ then the equations of motion (2.1) are invariant under the scale transformation $S$.

PROof. The result is easily verified by substitution in (2.1)-(2.6).

Theorem 7.11 implies that the equations of motion have a similarity solution, with unit speeds and $t$ replaced by the distance $\sigma=V t$ travelled along a path up to time $t$. This is a simple similarity, because $V$ is a constant of the motion.

From a different perspective, one easily writes (2.1) in terms of derivatives with respect to $\sigma$. The equations then describe the paths in differential geometry, rather than the motion. Since the paths are independent of speed, they apply to idealized mobiles of different types and to different distance scales.

The reduced equations (6.1)-(6.3) inherit this property. For example, (6.3) becomes

$$
\frac{d r_{R}}{d \sigma}=-2 \sin \frac{\phi_{R}}{2} \cos \zeta
$$

Then the constant $\phi_{R}$ appears in the equations, but $V$ does not.

\section{Planar solutions}

This section derives solutions for planar motion from equations (6.1)-(6.3), by exploiting the 2D symmetry. For planar motion, $\alpha=\pi / 2$ is fixed, and so (6.1) is redundant. Also $\boldsymbol{\Omega}_{F}$ is normal to the plane and has magnitude $W \sin \zeta$. The triangle formed by $V_{1}, V_{2}$ and $V_{R}$ shows that

$$
1-\eta^{2}=\frac{V_{R}^{2}}{4 V^{2}}=\sin ^{2} \frac{\phi_{R}}{2} .
$$


Thus (6.2) reduces to

$$
\dot{\zeta}=\frac{f V_{R}}{2 V^{2}}+\frac{V_{R}}{r_{R}} \sin \zeta .
$$

From (6.3), (7.1) and (8.1),

$$
\dot{M}=-\frac{f r_{R}}{2 V^{2}} \dot{r}_{R}
$$

Defining the indefinite integral

$$
I=\frac{1}{2 V^{2}} \int f r_{R} d r_{R}
$$

we have the following theorem.

THEOREM 8.1. If the motion is planar, then $M+I$ is a constant of the motion.

If $f=f_{p}$ then $I=\frac{1}{2} p r_{R}$. With (7.1) this gives the explicit solution

$$
r_{R}=\frac{2 c}{p+2 \sin \zeta}
$$

where $c$ is the constant value of $M+\frac{1}{2} p r_{R}$. Thus $r_{R}=2 c /(p+2)$ at CPA, and so the separation $\bar{r}_{R}$ at CPA is explicitly known for a given $p$. Conversely, $p$ may be chosen to achieve a given $\bar{r}_{R}$, and so one has advanced control over separation at CPA. This is valuable where safe separations are required. More explicitly,

$$
p=2 \frac{\bar{r}_{R}-r_{R}(0) \sin \zeta(0)}{r_{R}(0)-\bar{r}_{R}} .
$$

THEOREM 8.2. If $f=f_{p}$ and the motion is planar, then $\bar{r}_{R}$ increases with $p$.

This follows directly from (8.3). It justifies the name gain constant for $p$, and shows that $p$ provides consistent control over separation.

\section{Nonplanar solutions and invariant sets}

This section gives a partition of the reduced state space into invariant sets, and provides a complete reduction of the differential equations (6.1)-(6.3). The following notable result is crucial, where $\lambda=1 / \eta^{2}=(V / \bar{V})^{2}>1$.

THEOREM 9.1. In nonplanar motion $(\alpha \neq \pi / 2)$,

$$
A=M \frac{(\sin \alpha)^{\lambda}}{|\cos \alpha|^{\lambda-1}}
$$

is a constant of the motion.

Proof. Equations (6.1), (6.3) and (7.2) give

$$
\frac{d M}{d \alpha}=-M \frac{1-\eta^{2} \sin ^{2} \alpha}{\eta^{2} \sin \alpha \cos \alpha}=M\left(\tan \alpha-\frac{\lambda}{\sin \alpha \cos \alpha}\right)
$$


if $0<\alpha<\pi / 2$ or $\pi / 2<\alpha<\pi$. Formally integrating (9.2) gives

$$
\begin{aligned}
\log M & =-\log |\cos \alpha|-\lambda \log |\tan \alpha|+\log A \\
& =(\lambda-1) \log |\cos \alpha|-\lambda \log \sin \alpha+\log A,
\end{aligned}
$$

where the constant $A$ is determined by initial values of $M$ and $\alpha$. Also the theorem holds trivially if $\alpha=0$ or $\pi$.

Definition 9.2. In view of (7.1), the system (6.1)-(6.3) may be expressed in terms of the state variables $\alpha, M$ and $r_{R}$. Their domain $\left\{0 \leq \alpha \leq \pi, M>0, r_{R}>0\right\}$ defines a reduced state space $\Sigma_{R}$.

Corollary 9.3. $\Sigma_{R}$ comprises the following invariant subsets: $\mathrm{I}:\{0<\alpha<\pi / 2\}$, II: $\{\pi / 2<\alpha<\pi\}$, III: $\{\alpha=0\}$, IV: $\{\alpha=\pi\}$, and V: $\{\alpha=\pi / 2\}$.

Proof. From (9.1),

$$
M=\frac{A|\cos \alpha|^{\lambda-1}}{(\sin \alpha)^{\lambda}},
$$

which shows that $\sin \alpha$ cannot fall to zero while $r_{R}$ is finite, because $M \leq r_{R}$. Hence sets I and II are invariant. Sets III and IV are isolated, and so they are separately invariant. The solutions of Section 8 show that set $\mathrm{V}$ is invariant.

Remark. Sets III and IV, where $\sin \alpha$ is always zero, were identified after (5.17). These special solutions are described in Section 12.

Any motion in set I has a counterpart in set II which is its mirror image. Likewise, sets III and IV comprise mirror image pairs. The sets are illustrated in Figure 3. The $\cup$-shaped curves are plots of $M$ against $\alpha / \pi$, obtained from (9.3) with $\lambda=3$, where $\phi_{R}=\arccos (-1 / 3)=109.5^{\circ}$, with $A=1$ (labelled at $a$ and $\left.a^{\prime}\right), A=10(b$ and $\left.b^{\prime}\right), A=50\left(c\right.$ and $\left.c^{\prime}\right)$ and $A=200\left(d\right.$ and $\left.d^{\prime}\right)$.

The $\cap$-shaped curves are plots of $r_{R}$ against $\alpha / \pi$, obtained as follows. Equations (6.1), (6.3) and (7.1) give

$$
\frac{d r_{R}}{d \alpha}=\frac{V_{R}^{2} \sin \zeta}{2 f \eta^{2} \sin \alpha \cos \alpha}=\frac{V_{R}^{2} M}{2 r_{R} f \eta^{2} \sin \alpha \cos \alpha} .
$$

Substituting (9.3) herein gives

$$
\frac{d r_{R}}{d \alpha}=\frac{V_{R}^{2} A \lambda|\cos \alpha|^{\lambda-1}}{2 r_{R} f \cos \alpha(\sin \alpha)^{\lambda+1}} .
$$

This can be integrated to yield a relation $r_{R}=R(\alpha)$. Taking $f=f_{p}$ makes this relation explicit. For integer values of $\lambda$, the integration can be performed analytically, possibly due to a hidden symmetry. Taking $\lambda=3$ again, one gets

$$
r_{R}=r_{R}(0)-\frac{4 A}{3 p}\left(\frac{1}{\sin ^{3} \alpha}-1\right) .
$$




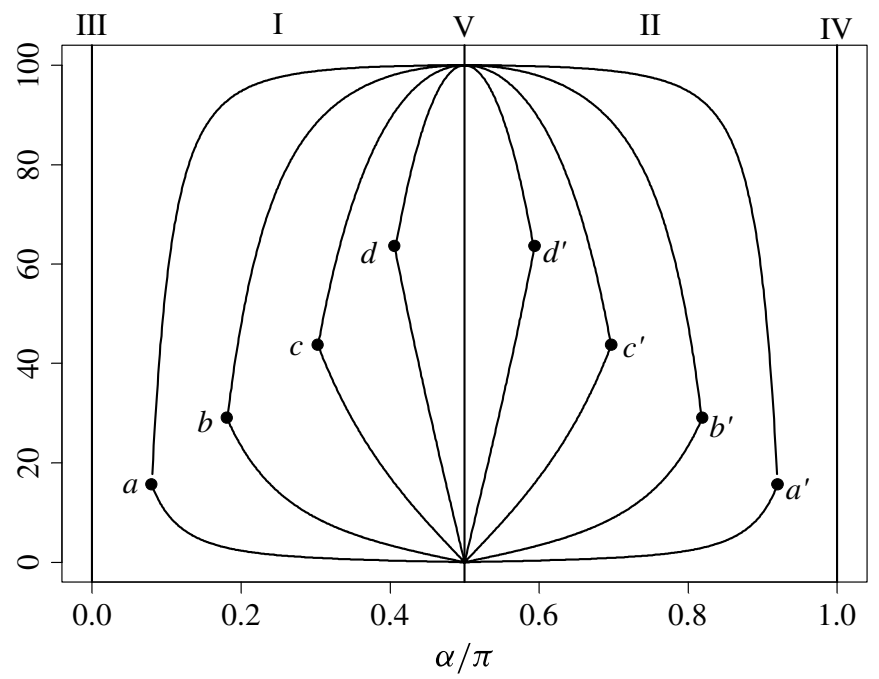

FIgURE 3. Plots of $M\left(\cup\right.$-shaped curves) and $r_{R}$ ( $\cap$-shaped curves) against $\alpha$, illustrating the invariant subsets I-V. The labelled dots are CPAs.

Choosing $p=1, r_{R}(0)=100$ and the above values of $A$ gives the $\cap$-shaped curves in Figure 3. The intersection points $a, b, \ldots$ in Figure 3 are the CPAs. Thus $M$ increases until it reaches CPA, and then reverses direction along the same curve in Figure 3. Likewise $r_{R}$ decreases until it reaches CPA, and then reverses direction.

The qualitative form of this evolution can be inferred in general from (6.1)-(6.3) and (7.1), as follows. In set I, (6.1) implies that $\dot{\alpha}<0$ when $\zeta<\pi / 2$, and $\dot{\alpha}>0$ when $\zeta>\pi / 2$. Hence $\alpha$ falls to a positive value at CPA, and thereafter increases until $\alpha=\pi / 2$ at UPE, where $M=0$ and $\zeta=\pi$.

Figure 3 shows that set $\mathrm{V}$ is unstable, in the sense that an initial state with a small $|\pi / 2-\alpha(0)|$ (that is nearly planar) will evolve so that $|\pi / 2-\alpha|$ increases until CPA. Figure 3 shows that this increase can be very large if $M(0)$ is small, implying a near collision course. The particles then achieve much better separation by diverging from the collision plane, which is intuitively plausible.

THEOREM 9.4. The equations of motion (6.1)-(6.3) can be reduced to quadrature.

Proof. Section 8 provides a proof for planar motion, and Section 12 for sets III and IV. For other nonplanar motion, (7.1) and (9.3) give

$$
\sin \zeta=\frac{M}{r_{R}}=\frac{A|\cos \alpha|^{\lambda-1}}{R(\alpha)(\sin \alpha)^{\lambda}},
$$

which implies a relation $\zeta=Z(\alpha)$. Substituting for $r_{R}$ and $\zeta$ in (6.1) gives an equation of the form $\dot{\alpha}=F(\alpha)$, which integrates to give an implicit relation $\alpha=G(t)$. Then $\zeta=Z[G(t)]$ and $r_{R}=R[G(t)]$, which completes the proof. 


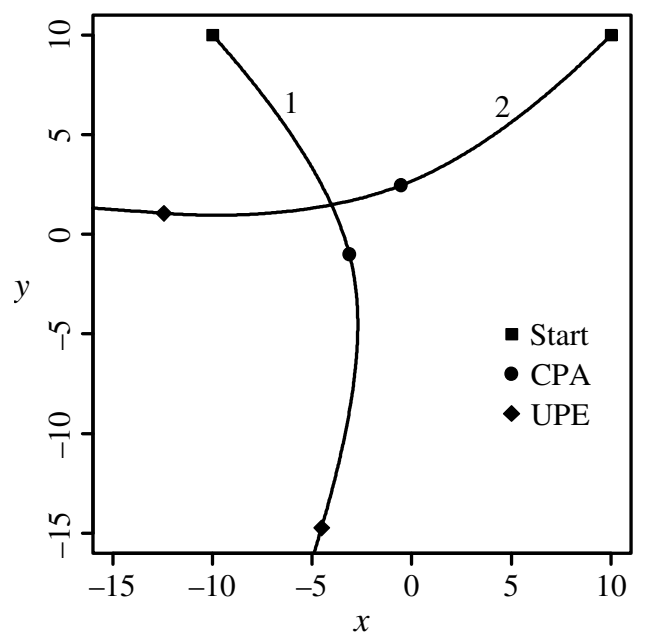

FIgure 4. Plan view of the 3D motion of two particles. Particle 1 turns upward (out of the page) and particle 2 turns downward.

REMARK. Each reduction step is implicitly associated with a one-parameter group of symmetries of the system [11, Theorem 2.66]. We have identified some of these groups, but there is scope for a more complete identification.

\section{Illustration of the motion}

Here we illustrate the 3D motion when $f=f_{p}$ and $p=0.5$. Fixed, orthogonal axes $x, y, z$ are chosen with $z$ pointing vertically up. Figure 4 is a plan view, or projection on the $(x, y)$ plane, of 3D paths. The distance scale is in arbitrary units, which could be chosen according to the application. Both particles are initially moving horizontally and particle 1 is slightly higher (0.03 units). Particles 1 and 2 make angles of $48^{\circ}$ and $-45^{\circ}$ with the $x$ axis, and so there is a small asymmetry in the initial conditions. By Theorem 7.11, the speeds do not affect the paths when $f=f_{p}$, and so they are not specified. Particle 1 climbs and particle 2 descends.

A notable feature of Figure 4 is the crossing of the projected paths. Planar motion (Section 8) produces similar paths [7]. Then particle (mobile) 2 crosses the path of particle 1, but safely behind particle 1. This allows effective separation without excessive turning, which is a vital feature of the motion and the guidance law. By contrast, simple Coulomb repulsion would tend to prevent crossing paths, and require severe rates of turn $[7,18]$ which might not be achievable by the mobiles.

Figure 5 is an elevation view, or projection on the $(y, z)$ plane, of the paths. Figure 6 is another elevation view, or projection on the $z, x$ plane. The projections exaggerate the steepness of the paths. The figures also show the CPA and the UPE. Figure 7 shows the paths projected normal to $\boldsymbol{r}_{R}$ at CPA, with $\boldsymbol{r}_{R}$ pointing into the page. Here $\boldsymbol{o}_{R}$ is the unit vector along $\boldsymbol{\Omega}_{R}$, and is orthogonal to $\boldsymbol{w}_{R}$. The $x^{\prime}$ axis is horizontal and normal to $\boldsymbol{r}_{R}$, while the $y^{\prime}$ axis is normal to the $x^{\prime}$ axis and $\boldsymbol{r}_{R}$. The projected paths are symmetric across $\boldsymbol{w}_{R}$ at CPA, which is consistent with Corollary 7.10. 


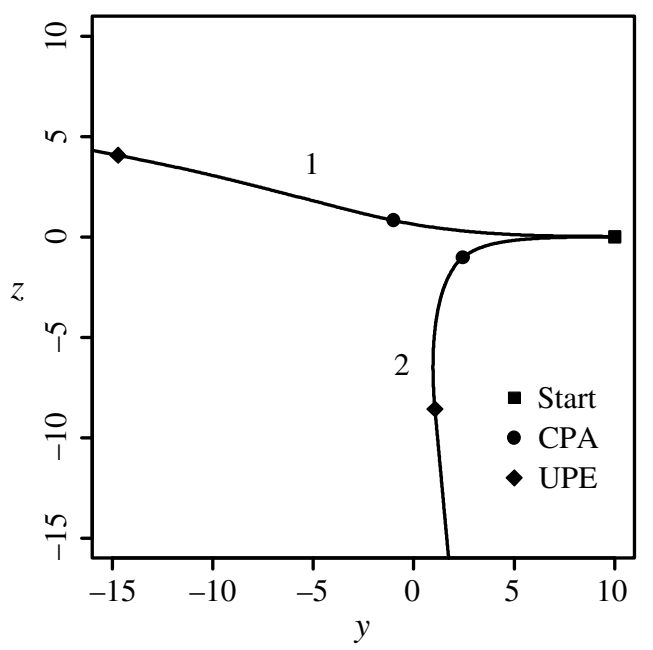

FIGURE 5. Side view of the 3D motion of the two particles from Figure 4.

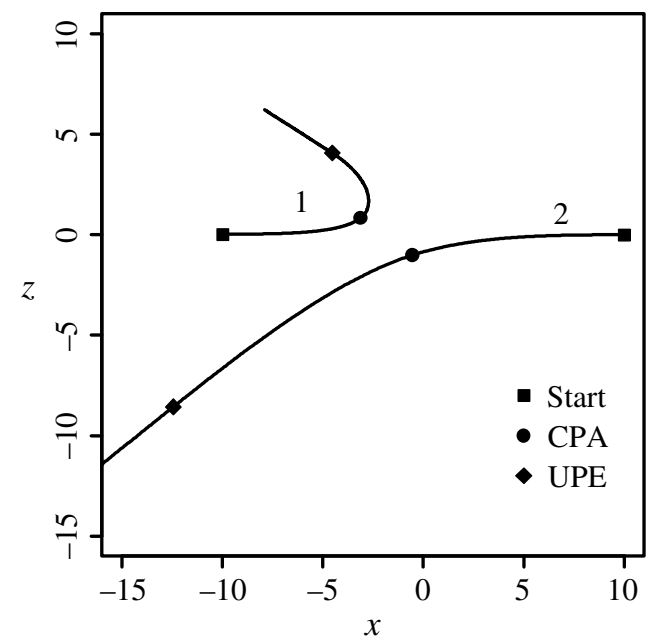

Figure 6. Front view of the 3D motion of the two particles from Figure 4.

The variation of $r_{R}$ and $M$ with $\sigma$ is shown in Figure 8, the variation of $\alpha$ with $\sigma$ is shown in Figure 9, and the variation of $\zeta$ with $\sigma$ is shown in Figure 10. These evidently have the symmetries described in Theorem 7.2, and belong to set II.

\section{Situation at CPA in 3D}

For safety's sake, it is important to give advanced warning of the ultimate proximity of the mobiles, and so one seeks to determine $r_{R}$ at CPA, as in the $2 \mathrm{D}$ formula (8.3). 


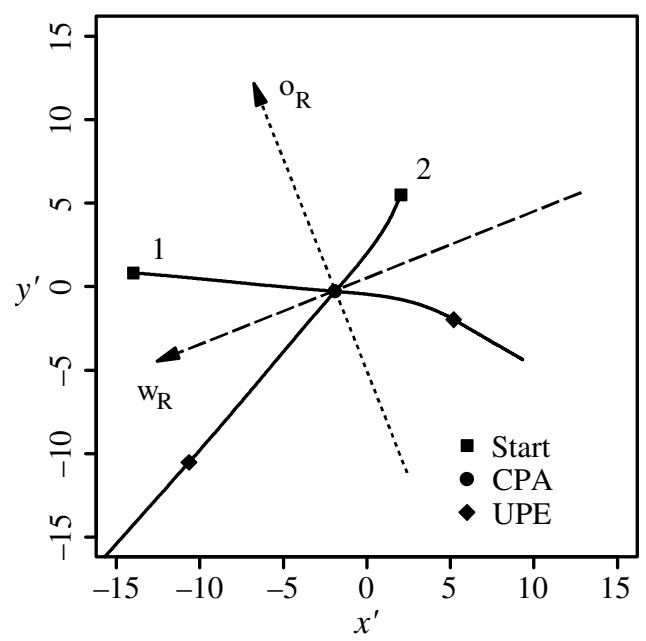

FIGURE 7. View of the 3D motion of the two particles from Figure 4, projected normal to $\boldsymbol{r}_{R}$ at CPA.

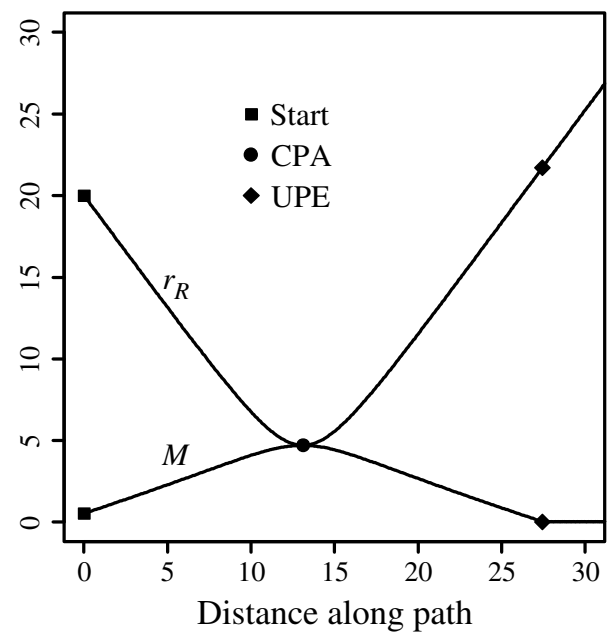

FIGURE 8. Change in separation $r_{R}$ and expected miss distance $M$ with distance $\sigma$ along a path, for the motion in Figure 4.

Since $M=r_{R}$ at CPA, one obtains from (9.3) and (9.4)

$$
R(\alpha)=\frac{A|\cos \alpha|^{\lambda-1}}{(\sin \alpha)^{\lambda}},
$$

where $r_{R}=R(\alpha)$ is obtained by integrating (9.4). Figure 3 illustrates this as an intersection of two curves. The equation can be solved numerically by Newton's method to determine $\alpha$ at CPA, say $\bar{\alpha}$. Since $R(\alpha)$ is given by an integral, it is 


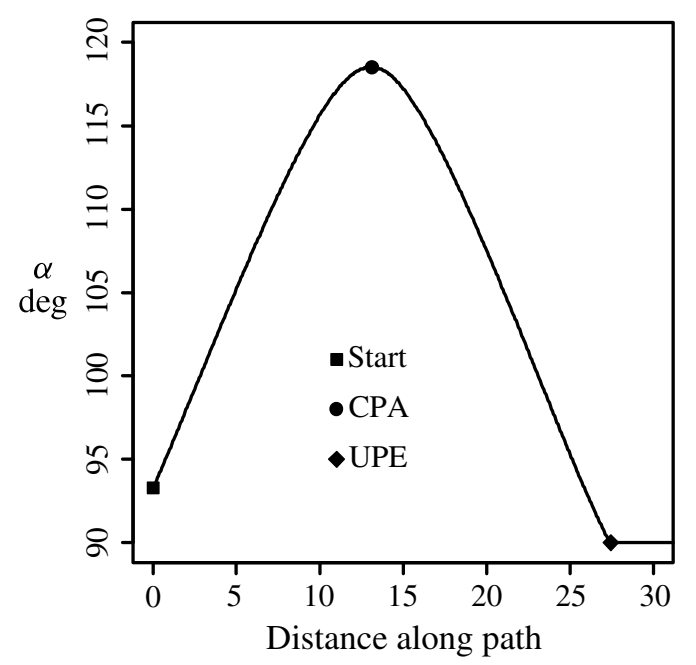

FIGURE 9. Change in state variable angle $\alpha$ with distance $\sigma$ along a path, for the motion in Figure 4.

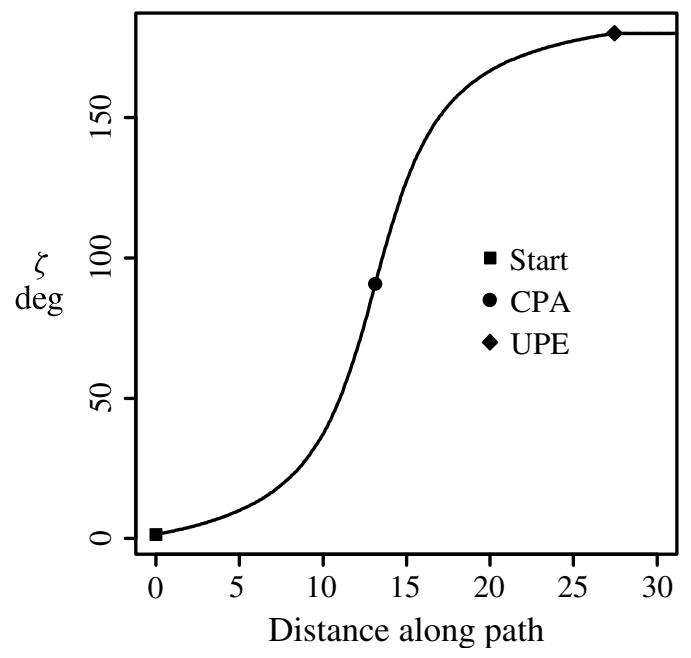

Figure 10. Change in state variable angle $\zeta$ with distance $\sigma$ along a path, for the motion in Figure 4 .

more efficient numerically to use a modified Newton method that reduces the number of integrations [4]. The separation $\bar{r}_{R}=R(\bar{\alpha})$ at CPA is an output of the numerical solution. In special cases like (9.5) one can obtain $\bar{\alpha}$ explicitly.

If $f=f_{p},(9.4)$ shows that $R(\alpha)$ depends on the gain constant $p$. Then Figure 11 shows the variation of $\bar{r}_{R}$ with $p$, for the example in Section 10. Larger $p$ yields a larger $\bar{r}_{R}$, but with diminishing returns as $p$ increases.

The general result is as follows, and extends Theorem 8.2 for set V. 


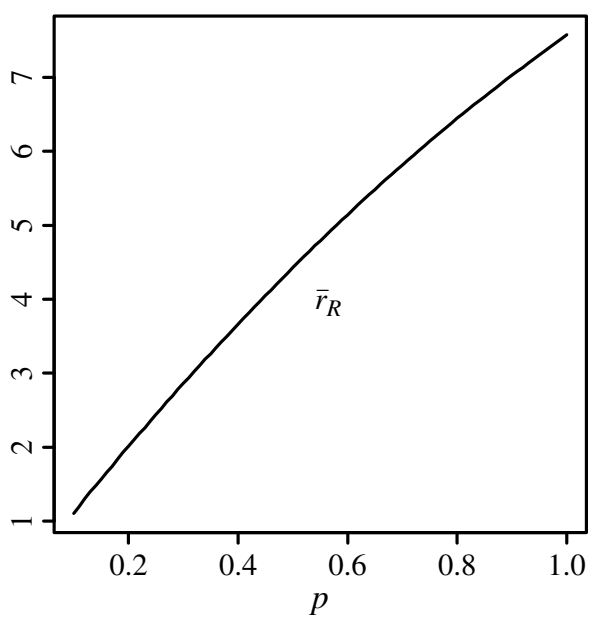

FIGURE 11. Relation between separation $\bar{r}_{R}$ at CPA and gain constant $p$, for the initial conditions in Figure 4.

THEOREM 11.1. If $f=f_{p}$ then $\bar{r}_{R}$ is an increasing function of $p$ in sets I and II.

Proof. In set II, where $\pi / 2<\alpha(0)<\bar{\alpha}$, (9.4) yields

$$
\bar{r}_{R}=r_{R}(0)-\frac{Z(\bar{\alpha})}{p},
$$

where

$$
Z(\bar{\alpha})=\frac{V_{R}^{2} A \lambda}{2 V^{2}} \int_{\alpha(0)}^{\bar{\alpha}} \frac{|\cos \beta|^{\lambda-2}}{(\sin \beta)^{\lambda+1}} d \beta .
$$

Then

$$
p=\frac{Z(\bar{\alpha})}{r_{R}(0)-\bar{r}_{R}} .
$$

Here $|\cos \bar{\alpha}|$ increases and $\sin \bar{\alpha}$ decreases as $\bar{\alpha}$ increases. It follows from (11.1) that $\bar{r}_{R}$ increases as $\bar{\alpha}$ increases. Now suppose that $\bar{r}_{R}$ increases for fixed $r_{R}(0)$ and $\alpha(0)$. Since $\bar{\alpha}$ increases and the integrand in (11.2) is positive, $Z(\bar{\alpha})$ increases. Then (11.3) implies that $p$ increases. Equivalently, $\bar{r}_{R}$ increases as $p$ increases. A similar argument applies to set I.

REMARK. The practical significance of this theorem is that one has consistent control of minimum separation through $p$. It also justifies the name gain constant for $p$. The result is extended to sets III and IV in Section 12.

In practice, a large $p$ could produce turning commands that exceed the capability of the mobile, as follows. Equation (7.3) shows that, at any instant of time, the mobiles have equal turn rates (3D angular speeds). This rate achieves a maximum at CPA. In practice, the turn rate of a mobile cannot exceed a given value. Then (7.3) sets an upper limit on $p$. If the mobiles are too close, and their paths are too convergent, then 
this could be inconsistent with (11.3) or (11.4), for a given $\bar{r}_{R}$. Then the nominated separation $\bar{r}_{R}$ could not be achieved, and a dangerous situation would exist. The above formulae therefore allow one to predict when such situations will arise.

Also of interest are the relative directions of the mobiles at CPA. One recalls (Theorem 4.1) that the paths make a constant angle $\phi_{R}$. As $\boldsymbol{V}_{R} \cdot \boldsymbol{r}_{R}=0$ at CPA, the directions make equal angles, $\gamma$ say, with $\boldsymbol{r}_{R}$ at CPA. As $\overline{\boldsymbol{V}}$ makes angle $\alpha$ with $\boldsymbol{r}_{R}$, one deduces, using (5.11), that

$$
\cos \gamma=\cos \frac{\phi_{R}}{2} \sin \bar{\alpha},
$$

which gives the 3D bearing of each mobile from the other at CPA. Thus $\gamma \geq \phi_{R} / 2$ in general. In planar motion, where $\alpha=\pi / 2,(11.4)$ reduces to $\gamma=\phi_{R} / 2$, as simple geometry confirms. For the symmetric case of Section 12, where $\sin \alpha=0$, (11.4) implies $\gamma=\pi / 2$ at CPA: then the mobiles are said to be "mutually at beam".

\section{Symmetric initial conditions and double helix motion}

This section analyses more symmetric, nonplanar behaviour. As mentioned, the alternative to (5.17) is $\sin \alpha=0$ for all $t$. The two cases $\alpha=0$ (invariant set III) and $\alpha=\pi$ (invariant set IV) are shown in Figure 3. If there is initially a height difference, these initial conditions hold if the initial velocities are horizontal and make equal internal angles with the $x$ axis. This admits any initial conditions in the two-dimensional invariant subset defined by $0<\zeta<\pi$ and $r_{R}>0$, and the motion is nontrivial.

Figure 12 illustrates the motion in this case. The initial conditions are as in Figure 4, except that the internal angles are both $45^{\circ}$. The projected paths are mirror images across the $y$ axis. General properties of the motion are summarized as follows.

THEOREM 12.1. For the symmetric initial conditions described, the forward paths of the particles are congruent in $E^{+}(3)$. The path segment of a particle up to CPA is congruent to the segment beyond CPA. Between EPG and UPE, the paths are helices of general type, and for constant $f$ the helices are circular. If $f=f_{p}$, the curvatures are $p / r_{R}$, independent of speed. Also $\boldsymbol{r}_{R}$ remains in a vertical plane, and Newton's third law holds.

Proof. For $\alpha=0$, (5.9) reduces to $\overline{\boldsymbol{V}}=\overline{\boldsymbol{V}} \boldsymbol{e}_{Z}$, and so $\overline{\boldsymbol{V}} \cdot \boldsymbol{r}_{R}=0$. Hence $\boldsymbol{V}_{1} \cdot \boldsymbol{r}_{R}=$ $-\boldsymbol{V}_{2} \cdot \boldsymbol{r}_{R}$, and so paths make equal internal angles with $\boldsymbol{r}_{R}$. The forward paths of the particles must then be congruent in $E^{+}(3)$. Then Corollary 7.10 implies that the path segment of a particle up to CPA is congruent to the segment beyond CPA.

In this case $\boldsymbol{V}_{R}$ is initially parallel with the $x$ axis. As $\boldsymbol{r}_{R}$ initially lies in the vertical plane, it follows that the fixed $z$ axis is horizontal and opposed to the $y$ axis. Now (5.8) implies $\boldsymbol{m} \perp \overline{\boldsymbol{V}}$, and so (4.3) implies $\chi=0$. Thus (4.2) implies that $\overline{\boldsymbol{V}}$ is constant, and so the $z$ axis of the frame $F$ is fixed. Moreover,

$$
\boldsymbol{V}_{i} \cdot \overline{\boldsymbol{V}}=V^{2} \cos ^{2}\left(\phi_{R} / 2\right)
$$




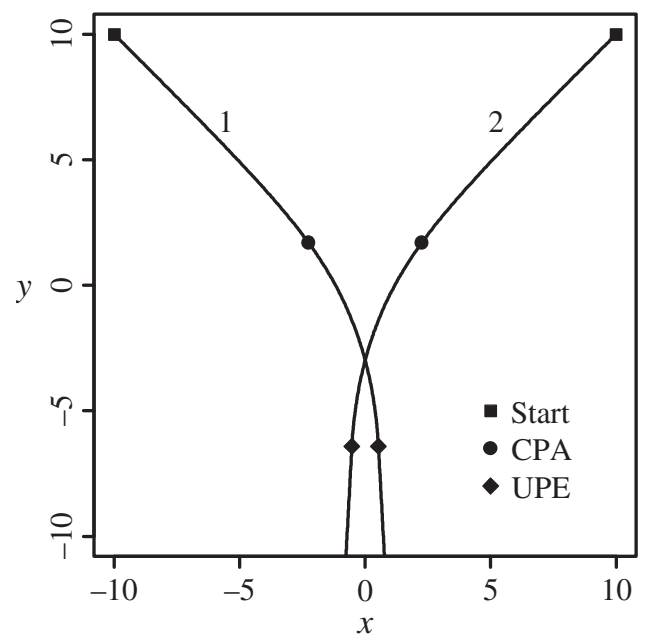

FIgURE 12. Plan view of the symmetric 3D motion of two particles. Particle 1 turns upward (out of the page) and particle 2 turns downward.

which is constant, and so each path makes a constant angle $\phi_{R} / 2$ with the fixed $z$ axis, forming a helix of general type [15]. From (7.3), the paths have equal instantaneous curvatures, which here reduce to $\kappa_{1}=\kappa_{2}=f / V^{2}$. The properties stated in the theorem then follow.

In the subsequent motion $Q=0$, and so (5.4) implies $\boldsymbol{\Omega}_{F}=\boldsymbol{\Omega}_{R}=-W \sin \zeta \boldsymbol{e}_{Z}$, which is along the $y$ axis. Thus $\boldsymbol{r}_{R}$ remains in a vertical plane. Both particles initially turn in a vertical plane, with 1 climbing and 2 descending. The constant $\overline{\boldsymbol{V}}$ implies that Newton's third law holds in this case. The argument for $\alpha=\pi$ is similar.

A few more details of the helices are as follows. According to (A.6), $J_{R}=0$, and so $\tau_{1}=\tau_{2}=f /\left[V^{2} \tan \left(\phi_{R} / 2\right)\right]$. Hence [15, Section 1.9] gives the pitch of both helices as $\kappa_{1} / \tau_{1}=\tan \left(\phi_{R} / 2\right)$, which is consistent with the above. For constant $f$, [15, p. 17] gives the radius of a circular cylinder containing a helix as

$$
a=\frac{\kappa}{\kappa^{2}+\tau^{2}}=\frac{V^{2}}{f} \cos ^{2} \frac{\phi_{R}}{2} .
$$

Hence the axis of the cylinder containing helix 1 is located at $\left(x_{1}(0), y_{1}(0)+a\right)$, and the axis of the cylinder containing helix 2 is located at $\left(x_{2}(0), y_{1}(0)-a\right)$. Thus the helices are not coaxial.

The equations for the relative motion simplify as follows. The $X$ and $Y$ components of (5.15) also imply that $\alpha$ is constant, and so (6.1) is redundant. If $f=f_{p},(6.2)$ reduces to

$$
\dot{\zeta}=\frac{V_{R}}{r_{R}}(\tilde{p}+\sin \zeta)
$$


where

$$
\tilde{p}=\frac{2 p V^{2}}{V_{R}^{2}}=\frac{p}{1-\cos \phi_{R}} .
$$

From (6.3) and (12.1),

$$
\frac{d r_{R}}{d \zeta}=-\frac{r_{R} \cos \zeta}{\tilde{p}+\sin \zeta}
$$

with solution

$$
r_{R}=\frac{c}{\tilde{p}+\sin \zeta} .
$$

This has the same form as the solution (8.2) for set V. Hence the relative motions in sets III and IV are the same, forming pairs with mirror image initial conditions, and are the same as in set $\mathrm{V}$ except for scale factors. Thus the properties described after (8.2) apply here too: in particular, $\bar{r}_{R}$ increases as $p$ increases. Combining this with Theorems 8.2 and 11.1 completes the picture.

THeOREM 12.2. If $f=f_{p}$, then $\bar{r}_{R}$ is an increasing function of $p$ in the whole state space $\Sigma_{R}$.

One notes that the case in Figure 4 differs from the present case only in the initial angle that mobile 1 makes with the $x$ axis, a difference of only $3^{\circ}$. A significant loss of symmetry occurs in Figure 4 because the initial state is close to a collision course $M=0$, which is intrinsically unstable, as noted after equation (2.6).

\section{Discussion}

The paper provides the first 3D solutions of a dynamical system concerning the important topic of preventing collisions, particularly midair collisions between aircraft. These enable one to predict or control the minimum separation, taking account of limitations on turn rates.

The method produces a surprising reduction of a seemingly intractable problem. It generates some unusual constants of the motion, such as $\bar{V}$ and (9.1). Some of the constants have arisen fortuitously, but it might be interesting to study their origins by identifying the associated symmetry groups. The equality of the curvatures (7.3) is another striking property, and is very much dependent on the particular form of the accelerations (2.2) and (2.3).

The artifice of using a rotating coordinate frame of reference $F$ for components of vectors might seem unpromising, but is particularly effective, partly because of the simple relation (5.17). As shown in the Appendix, the torsions reduce to surprisingly simple forms, starting from the rather unpromising definitions (A.1). All this appears to arise from fortuitous algebra and cancellations, but further analysis might clarify the simplicity.

Restricting the force coefficient $f$ to depend only on $r_{R}$ is more for simplicity than necessity. For example, many of the above results hold if we allow a function $f\left(r_{R}, M\right)$, such as that employed by Zeghal [18]. 
The steps in the reduction depend crucially on several imposed symmetries, particularly the fact that the particles are interchangeable. The transformation in Section 4 does not work if the particles have different speeds or different force coefficients. Nor is $\bar{V}$ a constant. The outstanding problem therefore is to make analytical progress in these cases. Some limited results of a qualitative kind were obtained in a previous paper [7].

\section{Acknowledgements}

The author thanks F. R. de Hoog for suggesting reference [4] and N. L. Fulton for helpful discussion of centroid motion.

\section{Appendix. Path torsions}

Here we derive the formulae (7.4)-(7.6) for the path torsions $\tau_{i}$. From [15, equations (5)-(5a) and (5) $-(5 b)]$, the torsions are given by

$$
\tau_{i}=\frac{\left(\boldsymbol{u}_{i} \dot{\boldsymbol{u}}_{i} \ddot{\boldsymbol{u}}_{i}\right)}{V^{3} \kappa_{i}^{2}},
$$

where the parenthetic term is the scalar triple product. From (2.1) and (2.2) we have $\dot{\boldsymbol{V}}_{1}=-f \overline{\boldsymbol{m}}_{1}$, and so

$$
\ddot{\boldsymbol{V}}_{1}=-\dot{f} \overline{\boldsymbol{m}}_{1}-f \dot{\boldsymbol{m}}_{1}
$$

which gives

$$
\dot{\boldsymbol{V}}_{1} \times \ddot{\boldsymbol{V}}_{1}=f^{2} \overline{\boldsymbol{m}}_{1} \times \dot{\overline{\boldsymbol{m}}}_{1}
$$

Therefore

$$
\left(\boldsymbol{u}_{1} \dot{\boldsymbol{u}}_{1} \ddot{\boldsymbol{u}}_{1}\right)=\frac{f^{2}}{V^{2}} \overline{\boldsymbol{m}}_{1} \cdot \dot{\overline{\boldsymbol{m}}} \times \boldsymbol{u}_{1}
$$

From (2.4),

$$
\dot{\bar{m}}_{1}=\dot{\boldsymbol{u}}_{1} \times\left(\boldsymbol{m} \times \boldsymbol{u}_{1}\right)+\boldsymbol{u}_{1} \times\left(\dot{\boldsymbol{m}} \times \boldsymbol{u}_{1}\right)+\boldsymbol{u}_{1} \times\left(\boldsymbol{m} \times \dot{\boldsymbol{u}}_{1}\right) .
$$

Since $\boldsymbol{u}_{1} \cdot \dot{\boldsymbol{u}}_{1}=0$, the first term becomes $-\boldsymbol{u}_{1} \dot{\boldsymbol{u}}_{1} \cdot \boldsymbol{m}$, and the last term becomes $-\boldsymbol{m} \cdot \boldsymbol{u}_{1} \dot{\boldsymbol{u}}_{1}$. Then (A.3) becomes

$$
\dot{\bar{m}}_{1}=\left(\boldsymbol{m} \cdot \dot{\boldsymbol{u}}_{1}\right) \boldsymbol{u}_{1}-\boldsymbol{u}_{1} \times \dot{\boldsymbol{m}} \times \boldsymbol{u}_{1}+\left(\boldsymbol{m} \cdot \boldsymbol{u}_{1}\right) \dot{\boldsymbol{u}}_{1} .
$$

The first and last terms contribute nothing to (A.2), and $\left(\boldsymbol{u}_{1} \times \dot{\boldsymbol{m}} \times \boldsymbol{u}_{1}\right) \times \boldsymbol{u}_{1}=\dot{\boldsymbol{m}} \times \boldsymbol{u}_{1}$. Hence (A.2) becomes

$$
\begin{aligned}
\left(\boldsymbol{u}_{1} \dot{\boldsymbol{u}}_{1} \ddot{\boldsymbol{u}}_{1}\right) & =-\frac{f^{2}}{V^{2}} \overline{\boldsymbol{m}}_{1} \cdot \dot{\boldsymbol{m}} \times \boldsymbol{u}_{1} \\
& =\frac{f^{2}}{V^{2}}\left(\boldsymbol{m}-\boldsymbol{u}_{1} \cdot \boldsymbol{m} \boldsymbol{u}_{1}\right) \cdot \dot{\boldsymbol{m}} \times \mathbf{u}_{1} \\
& =\frac{f^{2}}{V^{3}} J_{1}
\end{aligned}
$$


where

$$
J_{i}=\boldsymbol{m} \times \dot{\boldsymbol{m}} \cdot \boldsymbol{V}_{i} .
$$

By a similar argument $\left(\boldsymbol{u}_{2} \dot{\boldsymbol{u}}_{2} \ddot{\boldsymbol{u}}_{2}\right)=f^{2} J_{2} / V^{3}$. Putting these in (A.1) gives expressions of the form (7.4). To evaluate the $J_{i}$ we use (5.8) to obtain

$$
\dot{\boldsymbol{m}}=\dot{\zeta}(\cos \zeta,-\sin \zeta, 0)+\boldsymbol{\Omega}_{F} \times \boldsymbol{m} .
$$

Thus

$$
\begin{aligned}
\boldsymbol{m} \times \dot{\boldsymbol{m}} & =\dot{\zeta}\left(0,0,-\sin ^{2} \zeta-\cos ^{2} \zeta\right)+\boldsymbol{\Omega}_{F}-\boldsymbol{m} \boldsymbol{m} \cdot \boldsymbol{\Omega}_{F} \\
& =(0,0,-\dot{\zeta})+(Q, 0, W \sin \zeta)-(\sin \zeta, \cos \zeta, 0) Q \sin \zeta \\
& =\left(Q \cos ^{2} \zeta,-Q \sin \zeta \cos \zeta,-\dot{\zeta}+W \sin \zeta\right) .
\end{aligned}
$$

Since $\overline{\boldsymbol{V}}$ and $\boldsymbol{V}_{R}$ are known in $F$ components, it is convenient to write

$$
\boldsymbol{V}_{1}=\overline{\boldsymbol{V}}-\frac{1}{2} \boldsymbol{V}_{R} \quad \text { and } \quad \boldsymbol{V}_{2}=\overline{\boldsymbol{V}}+\frac{1}{2} \boldsymbol{V}_{R}
$$

Then (A.4) gives

$$
J_{1}=\bar{J}-\frac{1}{2} J_{R} \quad \text { and } \quad J_{2}=\bar{J}+\frac{1}{2} J_{R},
$$

where

$$
\bar{J}=m \times \dot{m} \cdot \bar{V}
$$

and

$$
J_{R}=\boldsymbol{m} \times \dot{\boldsymbol{m}} \cdot V_{R} .
$$

From (A.5), (5.9), (5.17) and (6.2) we get

$$
\begin{aligned}
\bar{J} & =\bar{V}\left[Q \cos ^{2} \zeta \sin \zeta \sin \alpha-Q \cos ^{2} \zeta \sin \zeta \sin \alpha+(-\dot{\zeta}+W \sin \zeta) \cos \alpha\right] \\
& =\bar{V}(-\dot{\zeta}+W \sin \zeta) \cos \alpha \\
& =-\bar{V} \frac{2 f}{V_{R}}\left(1-\eta^{2} \sin ^{2} \alpha\right) \cos \alpha
\end{aligned}
$$

which is (7.5). From (A.5), (5.1), (5.17) and (6.1) we get

$$
\begin{aligned}
J_{R} & =V_{R}\left(-Q \cos ^{3} \zeta-Q \sin ^{2} \zeta \cos \zeta\right) \\
& =-V_{R} Q \cos \zeta \\
& =-V_{R} \dot{\alpha} \\
& =-\frac{f \eta^{2} \cos \zeta}{\sin \zeta} \sin \alpha \cos \alpha,
\end{aligned}
$$

which is (7.6). 


\section{References}

[1] M. Alvarez and J. Llibre, "Heteroclinic orbits and Bernoulli shift for the elliptic collision restricted three-body problem", Arch. Ration. Mech. Anal. 156 (2001) 317-357; doi:10.1007/s002050100116.

[2] V. Barutello, D. L Ferrario and S. Terracini, "Symmetry groups of the planar three-body problem and action-minimizing trajectories", Arch. Ration. Mech. Anal. 190 (2008) 189-226; doi:10.1007/s00205-008-0131-7.

[3] A. M. Bloch, P. S. Krishnaprasad, J. E. Marsden and R. M. Murray, "Nonholonomic mechanical systems with symmetry", Arch. Ration. Mech. Anal. 136 (1996) 21-99; doi:10.1007/BF02199365.

[4] R. P. Brent, "A class of optimal-order zero-finding methods using derivative evaluations", in: Analytic computational complexity (ed. J. F. Traub), (Academic Press, New York, 1975) 59-73.

[5] E. Frazzoli, M. A. Dahleh and E. Feron, "Real-time motion planning for agile autonomous vehicles", J. Guid. Control Dyn. 25 (2002) 116-129.

[6] G. Fusco and W. M. Oliva, "Formation of symmetric structures in the dynamics of repelling particles", Arch. Ration. Mech. Anal. 151 (2000) 95-123; doi:10.1007/s002050050194.

[7] D. J. Gates, "Properties of a real-time guidance method for preventing a collision", J. Guid. Control Dyn. 32 (2009) 705-716; doi:10.2514/1.41197.

[8] R. Y. Gazit and J. D. Powell, "Aircraft collision avoidance based on GPS position broadcasts", Proc. 15th AIAA/IEEE digital avionics systems conference, Atlanta, GA, 1996 (IEEE, New York, 1996) 393-399; doi:10.1109/DASC.1996.559189.

[9] J. K. Kuchar and L. C. Yang, "A review of conflict detection and resolution modelling", IEEE Trans. Intelligent Transport Systems 1 (2000) 179-189; doi:10.1109/6979.898217.

[10] P. G. LeFloch and V. Shelukhin, "Symmetries and global solvability of the isothermal gas dynamics equations", Arch. Ration. Mech. Anal. 175 (2005) 389-430; doi:10.1007/s00205-004-0344-3.

[11] P. J. Olver, Applications of Lie groups to differential equations, 2nd edn (Springer, New York, 1993).

[12] G. W. Patrick, M. Roberts and C. Wulff, "Stability of Poisson equilibria and Hamiltonian relative equilibria by energy methods", Arch. Ration. Mech. Anal. 174 (2004) 301-344; doi:10.1007/s00205-004-0322-9.

[13] D. Rand, "Dynamics and symmetry. Predictions for modulated waves in rotating fluids", Arch. Ration. Mech. Anal. 79 (1982) 1-37; doi:10.1007/BF02416564.

[14] J. Sporrong and P. Uhlin, "System and method for avoidance of collision between vehicles" (Patent WO 01/46933 A1, June 28, 2001).

[15] D. J. Struik, Lectures on classical differential geometry, 2nd edn (Dover, New York, 1988).

[16] D. E. Swihart, B. Brannstrom, E. Griffin, R. Rosengren and P. Doane, "A sensor integration technique for preventing collisions between air vehicles", SICE 2002, Proc. 41st SICE Annual Conf. 1, Osaka, 2002 (SICE, Piscataway, NJ, 2002) 625-629; doi:10.1109/SICE.2002.1195481.

[17] L.-S. Wang, S.-J. Chern and C.-W. Shih, "On the dynamics of a tethered satellite system", Arch. Ration. Mech. Anal. 127 (1994) 297-318; doi:10.1007/BF00375018.

[18] K. Zeghal, "A comparison of different approaches based on force fields for coordination among multiple mobiles", Proc. IEEE/RSJ Intl. Conf. Intelligent Robots and Systems, Victoria, BC, 1998 (IEEE, Piscataway, NJ, 1998) 273-278; doi:10.1109/IROS.1998.724631. 